\title{
The Diagnostic Value of
} Endoscopic Balloon Catheter Usage for Detecting Early-Stage Primary Sclerosing Cholangitis in Endoscopic Retrograde Cholangiopancreatography: A Case Report

\author{
Burhan Ozdil ${ }^{a} \quad$ Arif Cosar $^{b} \quad$ Can Kece $^{c}$ Macit Sandikcia \\ Hikmet Akkiz \\ aDepartment of Gastroenterology, Faculty of Medicine, Cukurova University, \\ Adana, and Departments of ${ }^{b}$ Gastroenterology, and ${ }^{\mathrm{c}}$ Gastroenterological Surgery, \\ Research Hospital, Trabzon, Turkey
}

\section{Key Words}

Endoscopic retrograde cholangiopancreatography $\cdot$ Sclerosing cholangitis $\cdot$ Balloon catheter

\begin{abstract}
A 34-year-old woman was admitted to our clinic with abdominal pain, jaundice and pruritus. Endoscopic retrograde cholangiopancreatography was performed for cholestasis. Endoscopic retrograde cholangiopancreatography (ERCP) was judged as normal, after a standard ERCP cannula was used for the cholangiogram. However, marked canalicular irregularities were identified in cholangiography when pressurized contrast agent was administrated via balloon catheter. This cholangiographic view was thought to reveal an early-stage alteration of sclerosing cholangitis. Primary sclerosing cholangitis (PSC) is a chronic cholestatic liver disease characterized by destruction and fibrosis of the bile ducts. The diagnosis of PSC is based on typical cholangiographic findings, supported by nonspecific clinical signs and symptoms, cholestatic liver biochemical tests, and liver biopsy. Cholangiography is considered to be the gold standard for the diagnosis of PSC. The diagnosis is easy when diffuse multifocal biliary strictures, the hallmarks of the disease, resulting in a 'beaded' appearance on ERCP is detected. However, it may reveal a normal image in an early stage of the disease when bile duct changings are not prominent. We think that balloon catheter ERCP appears to facilitate the diagnosis of early-stage primary sclerosing cholangitis.
\end{abstract}




\section{Introduction}

Primary sclerosing cholangitis (PSC) is a chronic inflammatory condition and the disease's etiopathogenesis has not been well-defined. The etiology of PSC includes immune-mediated components and elements of undefined nature. The most common manifestations are fatigue, pruritus, jaundice and abdominal pain. PSC is characterized by progressive periductal obliterating fibrosis and strictures in the intrahepatic and extrahepatic bile ducts and usually follows a progressive course leading to cirrhosis with complications of portal hypertension and end-stage liver failure [1]. There is no proven medical treatment available for PSC and liver transplantation is currently the only lifeextending therapy for patients with end-stage disease [2]. The only population-based estimates of incidence and prevalence of PSC conducted in the United States revealed rates of 8.5-14.2 per 100,000 persons. Two-thirds of the cases are male [3]. Affected cases are generally young patients and the mean survival after diagnosis is approximately 12 years. Patients with PSC have an increased incidence of other autoimmune diseases [4]. More than $75 \%$ of the cases with PSC have ulcerative colitis. Five percent of the patients with ulcerative colitis have PSC $[5,6]$.

ERCP is considered to be the gold standard for the diagnosis of PSC. The characteristic cholangiographic abnormalities consist of multifocal strictures and dilatations in the intrahepatic and/or extrahepatic bile ducts. The increasing use of invasive cholangiography has led to diagnosing PSC in a high proportion of symptomatic patients; however, ERCP may occasionally reveal a normal image in an early phase. In the early stages of the disease, bile duct changings are not prominent [7]. Therefore, it may be possible to observe early biliary tree alterations by using balloon catheter while they usually may not be presented by standard cannulas in ERCP.

\section{Case Report}

A 34-year-old female patient who had complaints of postprandial nausea and vomiting for one month was admitted to our hospital with pruritus and icterus. The patient's height was $150 \mathrm{~cm}$, and her weight was $48 \mathrm{~kg}$. Weight loss of $8 \mathrm{~kg}$ and severe loss of appetite were determined in 6 months. In her medical history, she had complained of right upper quadrant pain and fever during the previous year. These findings had been misdiagnosed as urinary tract infection in a peripheral hospital, and she was administered antibiotics for the infection. The patient had a history of infertility. She had no history of medication except penicillin. Physical examination revealed icteric skin and conjunctiva. Laboratory results revealed AST: 350 U/l, ALT: 200 U/l, ALP: 353 U/l, GGT: 158 U/l, total protein: $6.1 \mathrm{~g} / \mathrm{dl}$, albumin: $3.3 \mathrm{~g} / \mathrm{dl}$, total bilirubin: $11.4 \mathrm{mg} / \mathrm{dl}$, direct bilirubin: $6.9 \mathrm{mg} / \mathrm{dl}$, prothrombin time: $31 \mathrm{~s}$, INR: 2.3, HBV (-), HCV (-), HAV (-), AMA (-), ANA (-), SMA (-), pANCA (+); test of occult blood in the feces was positive. The patient had no history of diarrhea. The previously performed ultrasonography for right upper quadrant pain and fever was normal at six months before. Ultrasonography revealed ascites, sludge in the gallbladder and chronic parenchymal liver changes at admission. No esophageal or gastric varices were detected on upper endoscopy. The patient underwent ERCP for cholestasis. Although the initial images were normal (fig. 1), later images, after pressurized contrast administration by balloon catheter, showed cotton thrown views and irregularities of canaliculs which resembled sclerosing cholangitis (fig. 2). Colonoscopy revealed normal findings. The patient underwent liver biopsy, and periductal fibrosis was observed which is an important marker of sclerosing cholangitis. The typical cholangiographic appearance of sclerosing cholangitis are diffuse multifocal biliary strictures and a 'beaded' appearance. These changings represent the advanced stage of the disease. The early-stage ERCP view is not known. In figure 1, a ERCP view by using a standard catheter is shown while balloon ERCP findings are shown in figure 2. In the balloon ERCP, canalicular irregularities and cotton thrown views are shown. 


\section{Discussion}

Diagnosis of primary sclerosing cholangitis is established by the detection of characteristic alterations in the biliary tree as seen with ERCP or percutaneous transhepatic cholangiography or by the detection of periductal fibrosis on liver biopsy. PSC is characterized by the presence of progressive periductal concentric, obliterative fibrosis and intrahepatic and/or extrahepatic bile duct strictures [8]. The characteristic pathological feature of PSC is concentric periductal 'onion-skin' fibrosis, which progresses to a narrowing and then obliteration of the intrahepatic small bile ducts. However, the role of liver biopsy in the evaluation of PSC appears to be of limited value, and only $10-15 \%$ of the cases may have this characteristic pathological finding [9]. Parenchymal findings are usually non-specific and should be interpreted along with clinical and radiological information, therefore ERCP is the gold standard. ERCP characteristically reveals segmental fibrosis of intrahepatic and/or extrahepatic bile ducts with saccular dilatation of normal intervening areas which results in the characteristic beads-on-a-string appearance $[10,11]$. However, these pathognomonic findings are seen in the advanced stage of the disease [12]. In the literature, an early-stage PSC finding is not available. An unremarkable ERCP is seen in $2 \%$ of all patients with typical clinical and/or biochemical and histological features of PSC; however, with normal findings on cholangiography this rare and unstudied condition is termed 'small-duct PSC' [13]. Liver biopsy is essential in patients in whom cholangiography is unremarkable for PSC. ERCP was performed in our case, because ALP, GGT and bilirubin levels were high. ERCP revealed a normal image of the intrahepatic and/or extrahepatic duct (fig. 1). The end branches were not filled well despite contrast administration with conventional cannulation technique. This condition may occasionally be observed in cases with normal biliary tree images. The end branches may not be filled properly due to the escape of the administered contrast agent through the ampulla orifice. According to our clinical experience, it can be an important problem not to fill the biliary tree with contrast agent due to previous sphincterotomy or decreased ampulla of Vater tonus. The biliary balloon is settled in a transpapillary position and after cannulation of the choledoch, it is inflated not to allow the escaping of contrast agent. Therefore the extrahepatic biliary tree can be filled with contrast agent better. In the early period of sclerosing cholangitis, characteristic findings such as consecutive contraction and expansion of the bile duct are not seen when using standard ERCP catheter. However, a number of minor changes in the bile duct begin as shown in this case. In our case, an image of atypical sclerosing cholangitis exhibiting involvement of minor bile ducts upon pressurized contrast administration via a biliary balloon catheter was demonstrated (fig. 2). For this reason, we performed liver biopsy. Typical findings of sclerosing cholangitis were determined. These cholangiographic findings in balloon ERCP are accepted as early stage of sclerosing cholangitis.

We think that balloon catheter ERCP may allow to fill all the end branches with high levels of ALP and GGT and appears to facilitate the diagnosis of early-stage primary sclerosing cholangitis. 
Fig. 1. The image obtained by a cannula reveals normal cholangiographic findings. The end branches are not further filled despite contrast administration.

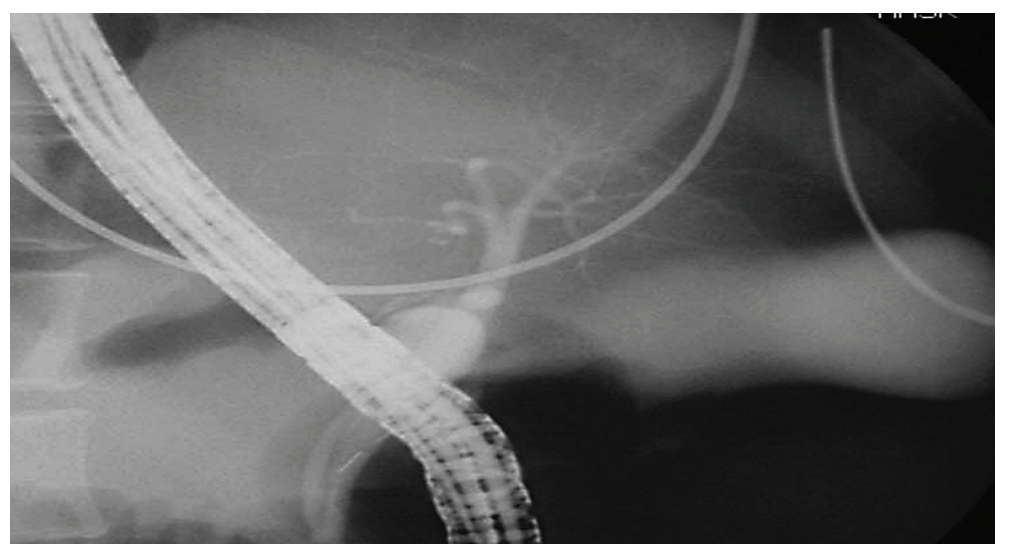

Fig. 2. Upon pressurized contrast administration by balloon catheter, thrown cotton view and irregularities are observed in the minor bile ducts.

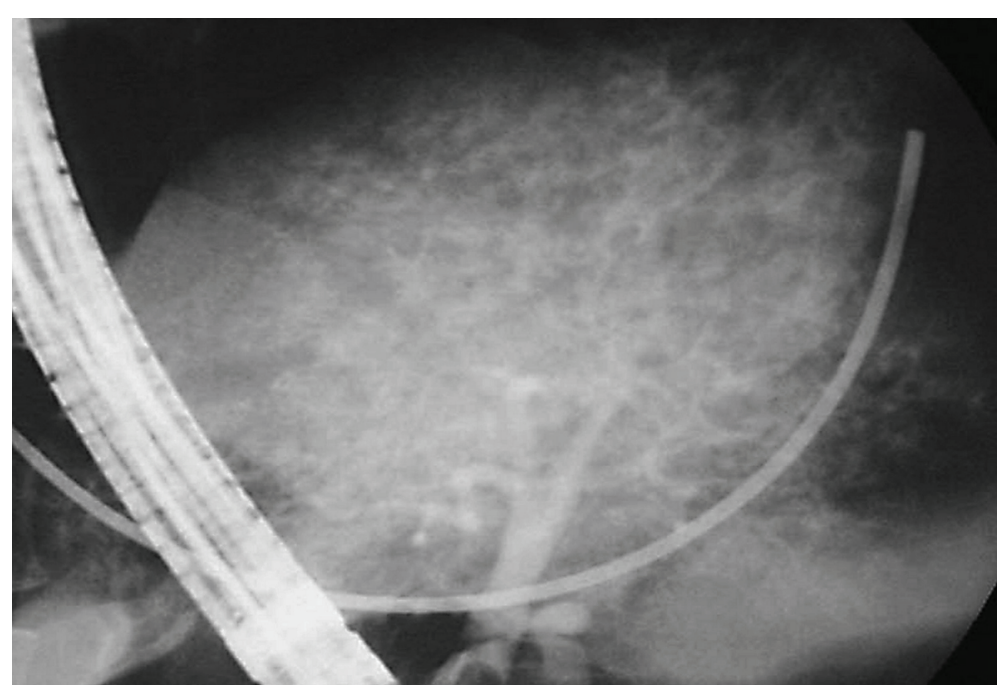




\section{References}

1 Silveira MG, Lindor KD: Primary sclerosing cholangitis. Can J Gastroenterol 2008;22:689-698.

-2 Cullen SN, Chapman RW: Review article: current management of primary sclerosing cholangitis. Aliment Pharmacol Ther 2005;21:933-948.

- 3 Broome U, Olsson R, Loof L, et al: Natural history and prognostic factors in 305 Swedish patients with primary sclerosing cholangitis. Gut 1996;38:610-615.

-4 Price $\mathrm{P}$, Witt $\mathrm{C}$, Allcock R, et al: The genetic basis for the association of the 8.1 ancestral haplotype (A1, B8, DR3) with multiple immunopathological diseases. Immunol Rev 1999;167:257-274.

5 Escorsell A, Pares A, Rodes J, et al: Epidemiology of primary sclerosing cholangitis in Spain. Spanish Association for the Study of the Liver. J Hepatol 1994;21:787-791.

-6 Aron JH, Bowlus CL: The immunobiology of primary sclerosing cholangitis. Semin Immunopathol 2009;31:383-397.

7 Björnsson E: Management of primary sclerosing cholangitis. Minerva Gastroenterol Dietol 2009;55:163-172.

$>8$ Gordon FD: Primary sclerosing cholangitis. Surg Clin North Am 2008;88:13851407.

9 Burak KW, Angulo P, Lindor KD: Is there a role for liver biopsy in primary sclerosing cholangitis? Am J Gastroenterol 2003;98:1155-1158.

10 Charatcharoenwitthaya P, Lindor KD: Primary sclerosing cholangitis: diagnosis and management. Curr Gastroenterol Rep 2006;8:75-82.

11 Wilschanski M, Chait P, Wade JA, et al: Primary sclerosing cholangitis in 32 children: clinical, laboratory, and radiographic features, with survival analysis. Hepatology 1995;22:1415-1422.

12 Worthington J, Chapman R: Primary sclerosing cholangitis. Orphanet Journal of Rare Diseases 2006;1:41.

13 Angulo P, Maor-Kendler Y, Lindor KD: Small-duct primary sclerosing cholangitis: a long-term follow-up study. Hepatology 2002;35:1494-1500. 